\title{
Time Domain Modeling and Characterization of Polymer Optical Fibers
}

\author{
Spiros Louvros, Athanassios C. Iossifides, Member, IEEE, George Economou, \\ George K. Karagiannidis, Senior Member, IEEE, Stavros A. Kotsopoulos, and Dimitris Zevgolis
}

\begin{abstract}
Polymer optical fibers (POFs) are of great interest the last decade, replacing glass fibers, in high-speed optical communication networks for short and medium distance applications. The characterization of POF as a transmission medium is of great importance when designing such optical networks. Based on a generalized impulse response model of multimode media, we developed a method for time bandpass response evaluation of a graded index polymer optical fiber (GIPOF). This model can be used to derive the response (in both time and frequency domains) of any arbitrary light source with given optical pulse characteristics.
\end{abstract}

Index Terms - Chromatic dispersion, differential mode attenuation, modal dispersion, polymer optical fibers (POFs).

\section{INTRODUCTION}

$\mathbf{P}$ OLYMER OPTICAL FIBER (POF) materials, including step-index as well as graded-index types, consist a strong candidate for future short distance communications, replacing conventional glass fibers, mainly because of easier transceivers' positioning and connectorization in addition to complete immunity to electromagnetic interference and radiation, and greater resistance to mechanical damage. Among the core POF materials, Polymethylmetacrylate (PMMA), seems to be one of the most effective with respect to attenuation, e.g., of $70-100 \mathrm{~dB} / \mathrm{km}$ in the region of $500-570 \mathrm{~nm}$ and $125-150 \mathrm{~dB} / \mathrm{km}$ at $650 \mathrm{~nm}$.

Existing analyses of POF transmission media, deal so far with the calculation of the input pulse broadening from the moments of the impulse response [1]-[3], or the evaluation of the frequency response [4], [5]. In this letter, we propose a full impulse response evaluation method. Though, due to lack of adequate measurement data we restrict the evaluation to the response of POF to a uniform (with respect to transmitted power per wavelength) source of finite linewidth (bandpass response). This response is adequate to characterize the transmission medium in the time domain, as soon as the evaluation bandwidth (linewidth) is greater than the arbitrary real source

Manuscript received February 28, 2003; revised October 7, 2003.

S. Louvros, G. Economou, and D. Zevgolis are with the Department of Physics, University of Patras, Patras 264 41, Greece (email: slouvros@cosmote.gr).

A. C. Iossifides is with COSMOTE S.A., Subdirection of Technical Matters, Thessaloniki, Greece (email: iossifides@the.forthnet.gr).

G. K. Karagiannidis is with Wireless Communications Group, Institute for Space Applications \& Remote Sensing, National Observatory of Athens, Athens, Greece (e-mail: gkarag@space.noa.gr).

S. A. Kotsopoulos is with University of Patras, Department of Electrical \& Computer Engineering, Wireless Telecommunications Laboratory, Patras, Greece (e-mail: kotsop@ee.upatras.gr).

Digital Object Identifier 10.1109/LPT.2003.822228 of interest. The response of the channel to this arbitrary source can be derived by direct convolution of the source (in time domain) with the calculated bandpass time response. Both chromatic (intramodal) and intermodal dispersions are taken into account. Differential mode attenuation (DMA) is included in the evaluation while mode coupling is not considered as it has been proven to be negligible for graded index polymer optical fiber (GIPOF) [4], [5].

\section{AnAlysis AND Evaluation Method}

\section{A. Proposed Model}

Consider a circular symmetric POF described by the refractive index profile [1]-[5]:

$$
n(r, \lambda)= \begin{cases}n_{1}(\lambda)\left[1-2 \Delta(\lambda)\left(\frac{r}{w}\right)^{\alpha}\right]^{1 / 2}, & 0<r<w \\ n_{1}(\lambda)[1-2 \Delta(\lambda)]^{1 / 2}, & r \geq w\end{cases}
$$

where $r$ is the offset distance from the core center, $\lambda$ is the freespace wavelength $\alpha$ is the core index exponent $(\alpha>0), n_{1}(\lambda)$ is the refractive index in the core center, $w$ is the core radius and $\Delta(\lambda)$ is the refractive index contrast. The refractive index of the cladding may be calculated as $n_{2}(\lambda)=n_{1}(\lambda)[1-2 \Delta(\lambda)]^{1 / 2}$.

The impulse response of POF, which is multimode in nature, can be described as a superposition of all excited modes of the $M(\lambda)$ mode groups, that is [1]

$$
h(t, z)=\sum_{m=1}^{M(\lambda)} h_{m}(t, z)
$$

with $h_{m}(\cdot)$ the impulse response of mode group $m$, given by [1]

$$
h_{m}(t, z)=\int_{0}^{\infty} A_{m}(\lambda, z) \delta\left[t-z T_{m}(\lambda)\right] d \lambda
$$

$A_{m}(\lambda, z)$ is the power of the modes in mode group $m$ at wavelength $\lambda$ and distance $z, \delta(\cdot)$ denotes the Dirac function and $T_{m}(\lambda)$ is the modal delay per unit length, given by [4], [5]

$$
\begin{aligned}
T_{m}(\lambda)=\frac{N_{1}(\lambda)}{c}[ & {\left[1-\frac{\Delta(\lambda)[4+\varepsilon(\lambda)]}{\alpha+2}\left(\frac{m}{M(\lambda)}\right)^{2 \alpha /(\alpha+2)}\right] } \\
& \cdot\left[1-2 \Delta(\lambda)\left(\frac{m}{M(\lambda)}\right)^{2 \alpha /(\alpha+2)}\right]^{-1 / 2}
\end{aligned}
$$

where $N_{1}(\lambda)=n_{1}(\lambda)-\lambda \cdot d n_{1}(\lambda) / d \lambda$ is the group index, $c$ is the speed of light in vacuum and $\varepsilon(\lambda)$ is the profile dispersion parameter given by

$$
\varepsilon(\lambda)=-\frac{2 \lambda n_{1}(\lambda)}{N_{1} \Delta(\lambda)} \frac{d \Delta(\lambda)}{d \lambda} .
$$


Since existing attenuation measurements are restricted in certain wavelength regions [6], we, instead of (3), calculate an equivalent bandpass response of the form

$$
r_{m}(t, z)=\int_{\lambda_{1}}^{\lambda_{2}} A_{m}(\lambda, z) \delta\left[t-z T_{m}(\lambda)\right] d \lambda .
$$

We embody, without loss of generality, variable $z$ in the modal delay function and drop it, since it has no effect in the following calculations. By setting $x=T_{m}(\lambda)$ in (6), we get

$$
\begin{aligned}
r_{m}(t) & =\int_{\lambda_{1}}^{\lambda_{2}} A_{m}(\lambda) \delta\left[t-T_{m}(\lambda)\right] d \lambda= \\
& =\int_{f_{m}\left(x_{1}\right)}^{f_{m}\left(x_{2}\right)} A_{m}\left(f_{m}(x)\right) \delta(t-x) f_{m}^{\prime}(x) d x
\end{aligned}
$$

since $\lambda=T_{m}^{(-1)}(x) \equiv f_{m}(x) \Rightarrow d \lambda=d f_{m}(x)=f_{m}^{\prime}(x) d x$, where $f_{m}^{\prime}(x)$ denotes the derivative of function $f_{m}(x)$ with respect to $x$ and $T_{m}^{(-1)}(\cdot)$ denotes inverse function.

Setting $B_{m}(x)=A_{m}\left(f_{m}(x)\right) f_{m}^{\prime}(x)$, (6) leads

$$
r_{m}(t)=\int_{f_{m}\left(x_{1}\right)}^{f_{m}\left(x_{2}\right)} B_{m}(x) \delta(t-x) d x
$$

which according to Dirac function properties simplifies to $r_{m}(t)=B_{m}(t)$. Thus, the impulse response of (2), reduces to

$$
r(t, z)=\sum_{m=1}^{M^{\prime}(t)} A_{m}\left[T_{m}^{(-1)}(t), z\right] \cdot \frac{d T_{m}^{(-1)}(t)}{d t}, \lambda_{1} \leq \lambda \leq \lambda_{2}
$$

that gives the response in terms of time delay and distance over a properly selected spectral width such as to explicitly include the source spectral width. $M^{\prime}(t)$ is the number of mode groups contributing to the response at instant $t$.

\section{B. Evaluation Method}

Evaluation of (9) begins with the declaration of an adequate evaluation region of wavelengths $\left[\lambda_{1}, \lambda_{2}\right]$ so as to include the source spectral width.

1) Inverse Modal Delay Function: For the evaluation region under consideration, the inverse modal delay function $T_{m}^{-1}(t)$ in (9), for each mode group, must be calculated. In doing so, the number of excited mode groups per wavelength is found by [5]

$$
M(\lambda)=2 \pi w \frac{n_{1}(\lambda)}{\lambda}\left(\frac{\alpha \Delta(\lambda)}{\alpha+2}\right)^{1 / 2}
$$

and the time response window of each mode group (the absolute time instants corresponding to wavelengths $\lambda_{1}$ and $\lambda_{2}$ ) is calculated. Two methods can then be used: either an inverse function per mode can be numerically calculated from (4), for the time samples under consideration; or a curve fitting method can be applied to the original modal delay function and axis inversion, resulting in one function per mode group, where, the time instant that the response at a specific wavelength appears, is declared. For a given time instant $t$ in the time response window, and for a specific mode group, the wavelength that results in a response at this instant is calculated using the inverse modal delay function. If this wavelength is in the evaluation region the attenuation of the mode is encountered in the response. If the wavelength is out of the evaluation region, the response is not taken into account. This procedure is repeated for the maximum number of excited mode groups $M(\lambda)$ in the evaluation wavelength region. In the response of a mode group, the response of the individual modes is summed, their number being calculated as the sum of all modes satisfying $m=2 \mu+v$, where $\mu$ and $v$ denote the radial and azimuthal nodes, respectively, of the mode [1].

2) Modal Attenuation: Originating from loss mechanisms, such as absorption, scattering, reflection, etc. modal attenuation can be described by $A_{m}(\lambda, z)=e^{-\gamma_{m}(\lambda) z}$, with $\gamma_{m}(\lambda)$ given by the empirical formula [5]

$$
\gamma_{m}(\lambda)=\gamma_{1}(\lambda)+\gamma_{1}(\lambda) I_{\rho}\left\{\eta\left[\frac{m}{M(\lambda)}-\frac{1}{M(\lambda)}\right]^{2 \alpha /(\alpha+2)}\right\}
$$

where $\gamma_{1}(\lambda)$ denotes the attenuation of the first mode, $I_{\rho}(\cdot)$ is the $\rho$ th-order modified Bessel of the first kind and $\eta$ is a weighting constant. $\rho$ and $\eta$ are calculated by fitting [5] to measured MDA profiles of [7], where a general MDA profile is given. In order to calculate the absolute attenuation for each mode we have to estimate $\gamma_{1}(\lambda)$. This may be done taking into account measurement results of [6], where the total attenuation coefficient $\Gamma(\lambda)$ of a monochromatically excited POF, is given. The total received power at a wavelength $\lambda$ equals the sum of individual received powers from all excited modes, that is,

$$
\sum_{m=1}^{M(\lambda)} e^{-\gamma_{m}(\lambda) z}=10^{-\Gamma(\lambda) z}
$$

For each mode in the mode group and for the wavelength producing a response to the evaluated time instant, the received power is found by (11) and normalization is applied so that (12) is verified.

With $T_{m}^{-1}(t)$ and $A_{m}\left[T_{m}^{(-1)}(t), z\right]$ given for each mode, evaluation of (9) is straightforward.

\section{RESULTS AND DISCUSSION}

A BEN-doped PMMA POF with a core radius $w=250 \mu \mathrm{m}$ at a center wavelength of $650 \mathrm{~nm}$ is considered in the following results. The refractive indexes of the core and the cladding materials follow a three-term Sellmeier function of wavelength with the oscillator strength and oscillator wavelength values as given in [2]. Uniform launching is assumed with equal initial power distribution of all the modes.

Considering a uniform (with respect to power per wavelength) source of root mean square (rms) bandwidth of 0.5 and $2 \mathrm{~nm}$, we have calculated in Fig. 1 the total rms time dispersion $\sigma_{\text {tot }}(z)$ of a 100-PMMA POF, in the presence (symbols $\mathbf{\Lambda}$ ) or not (symbols $\circ$ ) of DMA. Total dispersion may calculated by [1]

$$
\sigma_{\mathrm{tot}}(z)=\left[\frac{M_{2}(z)}{M_{0}(z)}-\tau^{2}(z)\right]^{1 / 2}
$$

where $\tau(z)=M_{1}(z) / M_{0}(z)$ is the mean delay time and $M_{n}(z)$ are the moments of the impulse response, given by [1]

$$
M_{n}(z)=\int_{0}^{\infty} t^{n} h(t, z) d t=\int_{0}^{\infty} t^{n} r(t, z) d t, \lambda_{1} \leq \lambda \leq \lambda_{2} .
$$




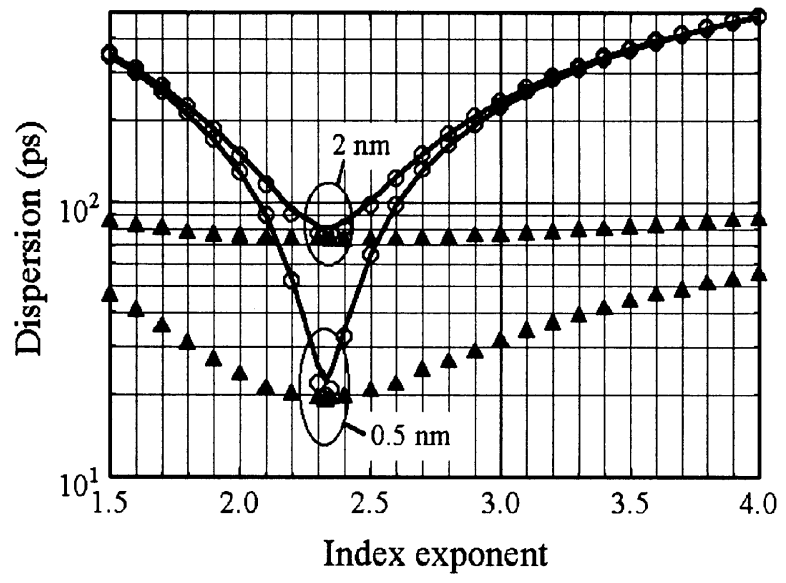

Fig. 1. Total dispersion of a $100 \mathrm{~m}$ long PMMA POF: (-) [5, eq. (17)] without DMA, (o) (14) without DMA, (ム) (14) with DMA.

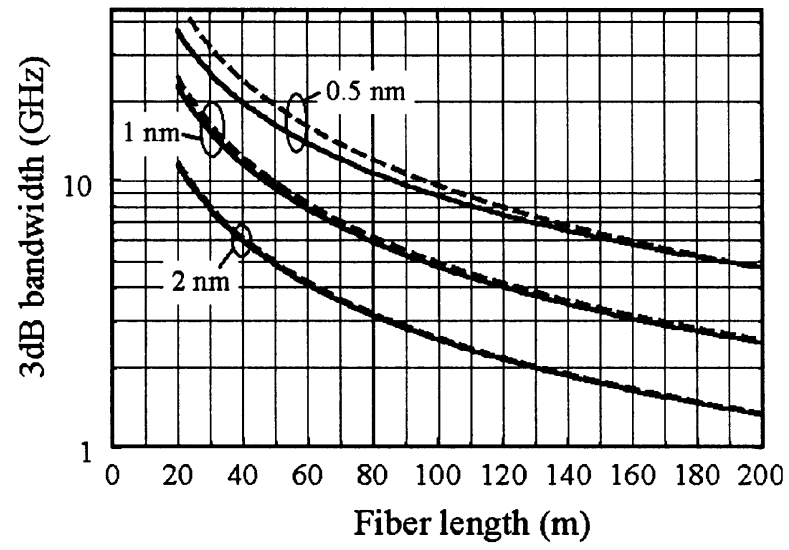

Fig. 2. 3-dB bandwidth of PMMA POF with different source spectral widths and for index exponents of (-) $a=2.1$ and (--) $a=2.33$.

The solid line in Fig. 1 (given for comparison) corresponds to the dispersion calculated through [5, eq. (17)], originating from [1], with no DMA. The results of the proposed method almost coincide with the ones of [5, eq. (17) ]. The insignificant difference in the region of lower dispersion (around the minimum) occurs because of the different evaluation methods and inaccuracies in the integrand evaluation of (14). On the other hand, it is evident that DMA reduces the dependence of total dispersion on the index exponent and this reduction is greater with increasing source bandwidth.

The frequency response of the medium can be evaluated with Fourier transform of the time response. Fig. 2 presents the $3-\mathrm{dB}$ bandwidth (DMA included) with respect to the length of the PMMA POF, for uniform sources with rms spectral width of 0.5 , 1 , and $2 \mathrm{~nm}$. With an index exponent of 2.33 (near optimum), the 3-dB bandwidth of a 100-m POF reaches 9.62, 4.62, and $2.56 \mathrm{GHz}$ for source spectral widths of $0.5,1$, and $2 \mathrm{~nm}$, respectively. The corresponding values are somewhat lower for an index exponent of 2.1, that is, 8.8, 4.76, and $2.53 \mathrm{GHz}$. In [5], $8.7 \mathrm{GHz}$ and $4.4 \mathrm{GHz}$ were predicted for $100 \mathrm{~m}$ and $200 \mathrm{~m}$ fiber

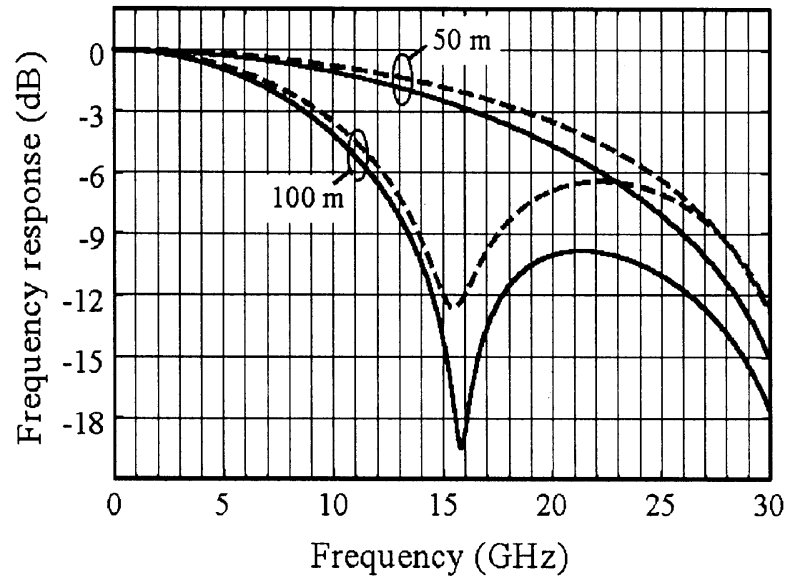

Fig. 3. Frequency response of 50- and 100-m PMMA long POF for index exponents of (-) $a=2.1$ and (- $) a=2.33$.

lengths with $a=2.1$ and a $0.5 \mathrm{~nm}$ wide source. The slight difference of our results ( 8.8 and $4.7 \mathrm{GHz}$, respectively) is due to the different evaluation methods, that is, no mode continuity is assumed and the overall attenuation is taken to be variable with wavelength in the proposed method, in contrast to [4]. It is concluded that the value of index exponent has not to be optimum in order to achieve high bandwidths, especially for long distances and wide sources (over 1-nm spectral width). The normalized frequency response of PMMA POF at 50 and $100 \mathrm{~m}$ excited by a 0.5 -nm wide source, is given in Fig. 3 where the 3 -dB bandwidths of both index exponents almost coincide. Though, the spectral characteristics (second lobe) are different and may be considered for source signal design.

The advantage of the proposed method lies in the exact time response provision for any source with known attenuation/ wavelength distribution. Thus, it can be used for time-domain source pulse shaping investigation and BER calculation with proper receiver modeling. So, it consists a method for end-to-end system modeling and evaluation of POF.

\section{REFERENCES}

[1] R. Olshansky and D. B. Keck, "Pulse broadening in graded-index optical fibers," App. Opt., vol. 15, no. 2, pp. 483-491, Feb. 1976.

[2] T. Ishigure, E. Nihei, and Y. Koike, "Optimum refractive-index profile of the graded-index polymer optical fiber, toward gigabit data links," Appl. Opt., vol. 35, no. 12, pp. 2048-2053, Apr. 1996.

[3] T. Ishigure, M. Satoh, O. Takanashi, E. Nihei, T. Nyu, S. Yamazaki, and Y. Koike, "Formation of the refractive index profile in the graded index polymer optical fiber for gigabit data transmission," J. Lightwave Tech., vol. 15, pp. 2095-2100, Nov. 1997.

[4] G. Yabre, "Comprehensive theory of dispersion in graded-index optical fibers," J. Lightwave Tech., vol. 18, pp. 166-177, Feb. 2000.

[5] — - "Theoretical investigation on the dispersion of graded-index polymer optical fibers," J. Lightwave Tech., vol. 18, pp. 869-877, June 2000 .

[6] W. Andreas, "Plastic optical fibers: Principles, components, installation," in Publicis MCD: Verlag, 1999.

[7] A. R. Mickelson and M. Eriksrud, "Mode-dependent attenuation in optical fibers," J. Opt. Soc. Amer., vol. 73, no. 19, pp. 1282-1290, Oct. 1983. 